\title{
TÉTANO GRAVE ASSOCIADO A CHOQUE SÉPTICO EM UMA PACIENTE IDOSA INTERNADA EM UNIDADE DE TERAPIA INTENSIVA
}

\section{SEVERE TETANUS ASSOCIATED WITH SEPTIC SHOCK IN AN ELDERLY PATIENT ADMITTED TO THE INTENSIVE CARE UNIT}

\author{
TÉTANOS GRAVE ASOCIADO CON CHOQUE SÉPTICO EN UNA \\ PACIENTE ANCIANA INGRESADA EN LA UNIDAD DE CUIDADOS \\ INTENSIVOS
}

\author{
Mayra Gonçalves Menegueti ${ }^{*}$ \\ Gil Cezar Alkmim-Teixeira ${ }^{* *}$ \\ JaCIARA MACHADO Viana** \\ Aníbal Basile-FILHo ${ }^{* * * *}$ \\ Olindo Assis Martins-Filho ${ }^{* * * *}$ \\ Maria Auxiliadora-Martins ${ }^{* * * * *}$
}

\begin{abstract}
RESUMO
Nós relatamos um caso de tétano grave associado a choque séptico em uma paciente de 61 anos admitida na Unidade de Terapia Intensiva (UTI) após perfurar o pé direito. A paciente apresentou perda de força muscular, paresia em membro inferior direito e disfagia. A paciente evoluiu com espasmos musculares generalizados, dispnéia e insuficiência respiratória. $\mathrm{O}$ espasmo intenso levou ao uso prolongado de ventilação mecânica invasiva, sedação e bloqueador neuromuscular. A evolução favorável deste caso está provavelmente relacionada aos avanços na gestão de UTI e uma equipe médica e de enfermagem bem treinadas.
\end{abstract}

Palavras chave: Tétano, choque séptico, idoso, Unidades de Terapia Intensiva.

\begin{abstract}
We report a case of severe tetanus associated with septic shock in a 61-year-old female admitted to the Intensive Care Unit (ICU) after perforating injury in the right foot. The patient presented with loss of muscle strength, paresis in the right lower limb and dysphagia. The patient's conditions worsened, progressing to generalized

\footnotetext{
*Enfermeira. Divisão de Terapia Intensiva, Departamento de Cirurgia e Anatomia, Hospital das Clínicas da Faculdade de Medicina de Ribeirão Preto, Universidade de São Paulo, Ribeirão Preto, SP, Brasil. Email: mayra_menegueti@yahoo.com.br ${ }^{* *}$ Médico. Divisão de Terapia Intensiva, Departamento de Cirurgia e Anatomia, Hospital das Clínicas da Faculdade de Medicina de Ribeirão Preto, Universidade de São Paulo, Ribeirão Preto, SP, Brasil. Email: gilteixeira@hotmail.com

${ }^{* * *}$ Médico. Divisão de Terapia Intensiva, Departamento de Cirurgia e Anatomia, Hospital das Clínicas da Faculdade de Medicina de Ribeirão Preto, Universidade de São Paulo, Ribeirão Preto, SP, Brasil. Email: jacimv@netsite.com.br

${ }^{* * * *}$ Médico. Divisão de Terapia Intensiva, Departamento de Cirurgia e Anatomia, Hospital das Clínicas da Faculdade de Medicina de Ribeirão Preto, Universidade de São Paulo, Ribeirão Preto, SP, Brasil. Email: abasile@fmrp.usp.br

${ }^{* * * * *}$ Biomédico. Laboratório de Biomarcadores de Diagnóstico e Monitoração, Instituto René Rachou, Fundação Oswaldo Cruz, Belo Horizonte, MG, Brasil. Email: oamfilho@cpqrr.fiocruz.br

${ }_{* * * * * *}$ Médico. Divisão de Terapia Intensiva, Departamento de Cirurgia e Anatomia, Hospital das Clínicas da Faculdade de Medicina de Ribeirão Preto, Universidade de São Paulo, Ribeirão Preto, SP, Brasil. Email: mamartins@hcrp.usp.br, mam_ martins@hotmail.com
} 
muscle spasms, dyspnea, and respiratory failure. Intense spasm made the prolonged use of invasive mechanical ventilation, sedation, and neuromuscular blockers necessary. The favorable outcome in the present case is probably related to advances in ICU management and well-trained medical and nursing staff.

Key words: Tetanus, shock, septic, elderly, Intensive Care Units.

\section{RESUMEN}

Se presenta un caso de tétanos relacionado con choque séptico grave en una paciente de 61 años ingresada en la Unidad de Cuidados Intensivos (UCI) después de perforar el pie derecho. El paciente tenía pérdida de fuerza muscular, parálisis de la pierna derecha y disfagia. El paciente desarrolló espasmos musculares generalizados, disnea e insuficiencia respiratoria. El espasmo intenso llevó al uso prolongado de ventilación mecánica, sedación y bloqueo neuromuscular. La evolución favorable de este caso está probablemente relacionada con los avances en la gestión de cuidados intensivos y un personal médico y de enfermería bien capacitado.

Palabras clave: Tétanos, choque séptico, anciano, Unidades de Cuidados Intensivos.

Fecha recepción: 31/03/10 Fecha aceptación: 09/12/11

\section{INTRODUÇÃO}

O tétano é uma doença infecciosa não contagiosa causada pela tetanospasmina, uma neurotoxina potente gerada pelo Clostridium tetani, uma bactéria gram-positiva anaeróbia, formadora de esporos (1-5). Apesar do tétano ser raro nos países desenvolvidos, continua a ser um importante problema de saúde pública em países em desenvolvimento. É ainda associada à elevada morbidade e mortalidade, apesar de ser facilmente prevenido por meio de vacina (6). A maioria dos casos de tétano ocorre em adultos que não são imunizados ou que não estão adequadamente imunizados (7-9). Estima-se que há cerca de um milhão de casos de tétano por ano em todo o mundo (10), com taxas de mortalidade variando de 10 a 53\% (11-14). Apesar da redução significativa na ocorrência de tétano, as taxas de mortalidade no Brasil permanecem acima dos $30 \%$, demonstrando a necessidade de um melhor planejamento e melhoria da qualidade de atendimento para este tipo de paciente (15).

O tétano tornou-se raro nos países desen- volvidos, graças a medidas profiláticas, bem como maior desenvolvimento socioeconômico e cultural. Isso tem permitido a imunização adequada da população e permitiu a prestação de cuidados satisfatórios aos pacientes traumatizados, bem como a adoção de procedimentos adequados para engravidar. No entanto, nem o bacilo foi erradicado do solo, nem a cadeia de transmissão da doença foi interrompida. Nestes países, o tétano ainda persiste nos idosos, devido à decomposição linear dos níveis séricos de antitoxina tetânica com o envelhecimento, para não mencionar a imunosenescência, que leva à diminuição da atividade dos linfócitos T auxiliares (16).

A presença de disfunção psicomotora no idoso facilita acidentes (17), e a baixa cobertura vacinal na população contribui para o aumento da mortalidade $(18,19)$. No Brasil, a distribuição etária do tétano tem sido pouco estudada. Um estudo anterior mostrou um declínio nas taxas de mortalidade em todas as faixas etárias, exceto para os idosos $(20,21)$. De fato, nas regiões norte e sul do Brasil a taxa de mortalidade desta doença aumentou em pessoas idosas. A introdução 
do tratamento de terapia intensiva e cuidados de enfermagem reduziu substancialmente a mortalidade, mas a sua taxa ainda é alta, particularmente nos idosos $(15,22,23)$.

O diagnóstico do tétano é clínico e reconhecido por contrações espasmódicas dos músculos do corpo. O trismo (espasmo do músculo masseter) é o primeiro sinal clássico do tétano, seguido por disfagia e alterações de voz. À medida que a doença progride, o paciente pode ter rigidez abdominal acompanhada de aumento da contração dos músculos da parede torácica e diminuição da amplitude de movimentos respiratórios, o que pode levar à insuficiência respiratória. Portanto, os cuidados de enfermagem, juntamente com toda a equipe multidisciplinar, tem um papel importante no monitoramento da permeabilidade das vias respiratórias, com especial atenção aos sinais clínicos de obstrução (24).

O objetivo deste estudo foi relatar um caso de tétano grave associado com choque séptico em uma paciente idosa internada na Unidade de Terapia Intensiva (UTI), detalhando a importância da assistência de enfermagem junto com a abordagem multidisciplinar para melhorar o prognóstico da evolução da doença.

\section{RELATO DE CASO}

Uma paciente de 61 anos, sexo feminino, residente na zona rural, foi admitida na UTI com sepse e insuficiência respiratória depois de um ferimento perfurante no pé direito. A paciente apresentava perda de força muscular, associada à paresia em membro inferior direito e disfagia. A condição da paciente se agravou com evolução para espasmos musculares generalizados, dispnéia e insuficiência respiratória, sendo transferida para a UTI. Após a admissão na UTI, a paciente desenvolveu piora da dispnéia e taquipnéia. Foi submetida à intubação orotraqueal e suporte ventilatório. $\mathrm{Na}$ admissão, o escore APACHE II para a paciente era 21 (risco de óbito de 35\%) (25). O exame físico revelou palidez cutânea, taquipnéia, utilização de musculatura acessória, com uma freqüência respiratória de $35 \mathrm{ipm}, \mathrm{SO}_{2}: 65 \%$, pressão arterial de $100 \times 70 \mathrm{mmHg}$, freqüência cardíaca de $105 \mathrm{bpm}$. A ausculta respiratória e cardíaca era normal. O exame do abdome foi normal, também. Os exames laboratoriais no momento da internação na UTI revelou creatinina $=1.0 \mathrm{mg} / \mathrm{dL}$, uréia $=48 \mathrm{mg} /$ $\mathrm{dL}$; bilirrubina total $=0,5 \mathrm{mg} / \mathrm{dl}$, sódio $=$ $136 \mathrm{mEq} / \mathrm{L}$, potássio $=3.6 \mathrm{mEq} / \mathrm{L}$; cálcio $=$ $1.24 \mathrm{mEq} / \mathrm{L}$, alanina aminotransferase ALT = 25; aspartato aminotransferase AST $=40$; albumina $=2,2 \mathrm{~g} / \mathrm{dl}$, hemoglobina $=11,9 \mathrm{~g} /$ $\mathrm{dL}$, hematócrito $=38 \%$; leucócitos $=12.000$ $/ \mathrm{mm}^{3}$ (bastonestes $=3 \%$, segmentados $=$ $79 \%$, e linfócitos $=18 \%$ ), plaquetas 286.000 $/ \mathrm{mm}^{3}$. A radiografia de tórax foi normal. As hemoculturas realizadas no primeiro dia de internação revelaram crescimento de Acinetobacter baumanii. Várias culturas de urina e culturas de escarro mostraram resultados negativos para microorganismos patogênicos.

A paciente foi tratada com imunoglobulina e toxóide tetânico, além do desbridamento da ferida e da administração de imunoglobulina no local da lesão. Além disso, a terapia com penicilina cristalina e metronidazol por via intravenosa foi iniciada. A sedação prolongada e bloqueadores neuromusculares permitiram o desenvolvimento de infecções hospitalares, como pneumonia, infecção do trato urinário, e infecção da corrente sanguínea relacionada ao cateter venoso central. A paciente também desenvolveu úlcera por pressão sacral grau III secundária à hospitalização prolongada, bem como a presença de desnutrição. A paciente tornou-se hemodinamicamente instável, e apesar da administração agressiva com fluidos, manteve hipotensão. Foi iniciado tratamento com droga vasoativa e antibiótico de largo espectro. A paciente recebeu alta da UTI após 92 dias de 
internação em boas condições. Atualmente, encontra-se clinicamente bem, consciente, e em acompanhamento ambulatorial sem seqüelas.

\section{DISCUSSÃO}

Apesar da redução na incidência de casos de tétano acidental no Brasil, não tem havido um decréscimo significativo da sua mortalidade. A centralização do atendimento prestado a esses pacientes em um único serviço especializado com o tratamento precoce em UTI tem sido decisiva para a redução da taxa de mortalidade (26). Este serviço conta com uma equipe médica e de enfermagem com vasta experiência, o que pode proporcionar melhor tratamento dos sintomas e prevenir complicações graves desta doença (22).

O tratamento na UTI resulta numa diminuição de quase quatro vezes a mortalidade quando comparado com pacientes tratados em enfermarias (27). A introdução do tratamento de terapia intensiva reduziu substancialmente a mortalidade, mas essas taxas ainda são altas, principalmente em idosos $(15,22)$. A prevenção da insuficiência respiratória e a manutenção da ventilação eficaz é melhor realizada em um ambiente de UTI. A intubação orotraqueal é muitas vezes necessária para proteger as vias aéreas, e a traqueostomia pode ser necessária para reduzir os estímulos que podem desencadear espasmos.

O diagnóstico do tétano é clínico. Poucas tentativas foram feitas para cultura do Clostridium tetani na prática clínica. Este bacilo é de difícil cultivo e pode estar presente na ausência de doença em pacientes imunocompetentes.

O tétano continua sendo uma ameaça em pessoas com imunização insuficiente. O tratamento de pacientes com tétano é um evento raro em países desenvolvidos, e a consciência é necessária para o reconhecimento dos sinais precoces desta doença rara, porém grave. De acordo com a literatura específica, o curso do tétano é tipicamente 3-4 semanas, embora possa ser maior nos casos mais graves $(2,3)$. Neste contexto, a admissão na UTI é muito importante para uma melhor evolução da doença, bem como a equipe médica e de enfermagem com experiência no tratamento de pacientes críticos sob ventilação mecânica (28).

A equipe de enfermagem na UTI tem um papel importante durante o atendimento ao paciente tetânico, identificando sinais clínicos e realizando intervenções para melhorar a condição do paciente (sistematização dos cuidados de enfermagem) (29).

O tétano pode evoluir para a insuficiência respiratória aumentando o risco de mortalidade e, portanto, requer constante monitoramento dos sinais vitais e padrão respiratório (30).

É importante que a equipe de enfermagem realize, quando necessário, a aspiração traqueobrônquica, a fim de manter as vias aéreas livres de secreções pulmonares, usando todas as precauções para evitar espasmos reflexos e acidentes, bem como infecções respiratórias.

O tétano também pode levar à redução da mobilidade física, o que propicia o aparecimento de lesões de pele aumentando o risco de infecção, como ocorreu com nossa paciente. Portanto, é de fundamental importância o treinamento dos profissionais de enfermagem para prevenir e tratar as úlceras por pressão.

Outro papel importante da equipe de enfermagem é realizar a manutenção de um ambiente tranquilo, garantindo o uso de tampões para os ouvidos a fim de minimizar o ruído durante a prática de enfermagem. Além disso, o controle da luminosidade é importante, pois assim como o ruído, este estímulo pode induzir espasmos musculares e contrações que geralmente pioram o curso da doença.

Ressaltamos a importância da cobertura vacinal e chamamos a atenção para a condi- 
ção precária da aplicação de profilaxia tetânica em cuidados hospitalares voltados para os idosos.

Em conclusão, o resultado favorável no presente caso, provavelmente está relacionado aos avanços na gestão de UTI e uma equipe médica e de enfermagem bem treinadas. No entanto, a longa permanência destes pacientes na UTI pode aumentar a incidência de complicações infecciosas.

Agradecimentos: Nós agradecemos a Fundação de Amparo ao Ensino, Pesquisa e Assistência (FAEPA) do Hospital das Clínicas e Faculdade de Medicina de Ribeirão Preto-USP pelo apoio.

Conflito de interesse: Todos os autores não possuem conflito de interesse.

\section{REFERÊNCIAS}

1. Bleck TP. Tetanus. En: Scheld WM, Whitney RJ, Durack DT, editores. Infections of the central nervous system. New York: Raven Press. 1991: 603-24.

2. Horn J, Vroom MB, Tijssen MA, Schultz MJ. Two cases of tetanus? J Intensive Care Med. 2006; 21(6): 364-8.

3. Veronese R, Focaccia R, Tavares W, Mazza CC. Tétano. En: Veronesi R, editor. Doenças Infecciosas e parasitárias. 8va ed. São Paulo: Guanabara Koogan; 1991. p. 447-466.

4. Bartlett JG. Tetanus. En: Goldman L \& Ausiello D, editores. Cecil textbook of Medicine. 22 ed. New York: WB Saunders; 2004. p. 1841-1842.

5. Abrutyn E. Tetanus. En: Isselbacher KJ, Braunwald E, Wilson JD, et al. Harrison's principles of internal medicine. 15 ed. New York: McGraw-Hill; 2001. p. 918920.

6. Fauveau V, Mamdani M, Steinglass R, Koblinsky M. Maternal tetanus: magni- tude, epidemiology and potential control measures. Int J Gynaecol Obstet. 1993; 40(1): 3-12.

7. Bleck TP. Clostridium tetani (Tetanus). En: Mandell GL, Bennett JE \& Dolin R, ed. Principles and practice of infectious diseases. 6ta ed. New York: Churchill Livingstone; 2005. p. 2817-2821.

8. Dundar V, Yumuk Z, Ozturk-Dundar D, Erdogan S, Gacar G. Prevalence of tetanus immunity in the Kocaeli Region, Turkey. Jpn J Infect Dis. 2005; 58(5): 279-282.

9. Habib AG. Tetanus complicating snakebite in Northern Nigeria: clinical presentation and public health implication. Acta Trop. 2003; 85(1): 87-91.

10. Miranda-Filho DB, Ximenes RAA, Bernardino SN, Escariãi AG. Identification of risk factors for death from tetanus in Pernambuco, Brazil: a casecontrol study. Rev Inst Med Trop Sao Paulo. 2000; 42(6): 333-339.

11. Edmondson RS, Flowers MW. Intensive care in tetanus: management, complications, and mortality in 100 cases. Br Med J. 1979; 1(6175): 1401-4.

12. Miranda-Filho DB, Ximenes RA, Barone AA, Vaz VL, Vieira AG, Albuquerque VM. Randomised controlled trial of tetanus treatment with antitetanus immunoglobulin by the intrathecal or intramuscular route. BMJ. 2004; 328(7440): 615-617.

13. Saltoglu N, Tasova Y, Midikli D, Borgut R, Dündar IH. Prognostic factors affecting deaths from adult tetanus. Clin Microbiol Infect. 2004; 10(3): 229-33.

14. Amare A, Yami A. Case-fatality of adult tetanus at Jimma University Teaching Hospital, Southwest Ethiopia. Afr Health Sci. 2011; 11(1): 36-40.

15. Brauner JS, Vieira SR, Bleck TP. Changes in severe accidental tetanus mortality in the ICU during two decades in Brazil. Intensive Care Med. 2002; 28(7): 930-5.

16. Kishimoto S, Tomino S, Mitsuya H, Fujiwara H, Tsuda H. Age-related decline in the in vitro and in vivo syntheses of 
anti-tetanus toxoid antibody in humans. J Immunol. 1980; 125(5): 2347-52.

17. Burnside IM. Enfermagem e os idosos. São Paulo: Andrei; 1979.

18. Thwaites CL, Yen LM, Glover C, Tuan PQ, Nga NTN, Parry J et al. Predicting the clinical outcime of tetanus: the tetanus severity score. Trop Med Int Health. 2006; 11(3): 279-87.

19. Lima VMSF, Garcia MT, Resende MR, Nouer SA, Campos EOM, Papaiordanou PMO et al. Tétano acidental: análise do perfil clínico e epidemiológico de casos internados em hospital universitário. Rev Saude Publica. 1998; 32(2): 166-71.

20. Fundação Nacional de Saúde. Boletim Epidemiológico: Evolução temporal das doenças de notificação compulsória no Brasil de 1980 a 1998. Centro Nacional de Epidemiologia, Ministério da Saúde, Brasília, 1999.

21. Secretaria de Vigilância em Saúde. Guia de Vigilância Epidemiológica. 6a edição. Ministério da Saúde, Brasília, 2005.

22. Gouvêa PAC, Silva CE, Miranda Filho DdeB, Bernardino SN, Escarião AG, Ximenes RA, et al. Mortality trend due to accidental tetanus from 1981 to 2004 in Pernambuco and analysis of the impact on intensive care unit attendance. Rev Soc Bras Med Trop. 2009; 42(1): 54-57.
23. Moraes EN, Pedroso ER. Tétano no Brasil: doença do idoso? Rev Soc Bras Med Trop. 2000; 33: 271-275.

24. Pagliuca LMF, Feitosa AR, Feijão AR. Tétano na população geriátrica: problemática na saúde coletiva? Rev Lat Am Enfermagem. 2001; 9(6): 69-75.

25. Knaus WA, Draper EA, Wagner DP, Zimmerman JE. APACHE II: A severity of disease classification system. Crit Care Med. 1985; 13(10): 818-29.

26. Greco JB, Neto JT, Júnior JBC. Accidental tetanus: prognosis evaluation in a historical series at a hospital in Salvador, Bahia, Brazil. Rev Inst Med Trop Sao Paulo. 2003; 45(1).

27. Kefer MP. Tetanus. Am J Emerg Med. 1992;10(5): 445-8.

28. Chalya PL, Mabula JB, Dass RM, Mbelenge N, Mshana S, Gilyoma JM. Ten-year experiences with Tetanus at a Tertiary hospital in Northwestern Tanzania: A retrospective review of 102 cases. World J Emerg Surg. 2011; 6:20.

29. Smeltzer SC, Bare BG. Brunner/ Studarth: Tratado de Enfermagem Médico Cirúrgica. $7 \mathrm{ma}$ ed. Rio de Janeiro: Guanabara Koogan; 1994.

30. Lee HC, Ko WC, Chuang YC. Tetanus of the elderly. J Microbiol Immunol Infect. 2000; 33(3): 191-6. 